\title{
Optical Quality and Tear Film Analysis Before and After Intranasal Stimulation in Patients with Dry Eye Syndrome
}

\author{
Samuel F Passi ${ }^{1,2}$ \\ Cassandra C Brooks (D) \\ Atalie C Thompson (D) \\ Preeya K Gupta $\mathbb{D}^{\prime}$ \\ 'Department of Ophthalmology, Duke \\ University, Durham, NC, USA; \\ ${ }^{2}$ Minnesota Eye Consultants, \\ Bloomington, MN, USA
}

This article was published in the following Dove Press journal: Clinical Ophthalmology

\begin{abstract}
Purpose: To evaluate the optical quality and tear film quality in patients with dry eye syndrome (DES) before and after using an intranasal neurostimulation device to stimulate tear production.
\end{abstract}

Methods: Cross-sectional review of 33 eyes in 21 patients with DES who underwent optical quality and tear film analysis as part of their routine dry eye evaluation pre- and postneurostimulation trial in office. Optical quality was assessed by measuring the objective scattering index (OSI) and modulation transfer function (MTF). The time to blink and mean tear film OSI were used to analyze the tear film.

Results: Optical quality after the use of the intranasal neurostimulator improved with an average decrease (improvement) in OSI of $0.30 \pm 0.68(\mathrm{P}=0.015)$ and an average increase (improvement) in MTF of $2.12 \pm 9.2(\mathrm{P}=0.15)$. Pre-intranasal neurostimulation OSI had a positive correlation with age (Spearman's rho 0.60, p < 0.001), while MTF had a negative correlation (Spearman's rho $-0.38, \mathrm{p}=0.03$ ). Pre-intranasal neurostimulation OSI had a negative correlation with MTF (Spearman's rho $-0.85, \mathrm{p}<0.001$ ) and a positive correlation with tear film mean OSI (Spearman's rho 0.85, $\mathrm{p}<0.001$ ).

Conclusion: The optical quality of patients with DES is improved with the use of intranasal neurostimulation for tear production, and there was a trend towards improved MTF though not statistically significant. Future studies are needed to follow patients longitudinally.

Keywords: neurostimulation, objective scatter index, modulation transfer function, dry eye syndrome

\section{Introduction}

Dry eye syndrome (DES) is one of the most common reasons patients visit an eye care provider in the United States. ${ }^{1}$ The prevalence of symptomatic DES in one US study was found to be $14.6 \%{ }^{2}$ The cumulative 10 -year incidence of DES in a separate study by Moss et al was calculated to be $21.6 \%{ }^{3}$ DES is important to recognize not only because of its negative impact on patient comfort but also due to its detrimental effect on optical quality parameters, such as objective scatter index (OSI) and modulation transfer function (MTF). ${ }^{4-6}$ Historically, DES diagnostics were limited to only a few tests, and treatment was mostly limited to artificial tears and warm compresses. However, in response to the massive influx of DES patients, there has been tremendous growth in both the diagnostic and therapeutic approaches to DES. From a diagnostic standpoint, point of care testing with tear osmolarity and tear matrix metalloproteinase-9 (MMP-9) have helped identify
Correspondence: Preeya K Gupta Email preeya.gupta@duke.edu 
patients with a large inflammatory component to their DES. Further, meibography has allowed a more detailed assessment of the state of the meibomian glands. From a therapeutic standpoint, we now have a potpourri of artificial tear and ointment options, anti-inflammatory drops such as cyclosporine and lifitegrast, intense pulsed light (IPL), and thermal pulsation for meibomian gland disease. ${ }^{7}$ In many cases, DES is multifactorial and until recently all treatments were only able to target one component of the DES. For example, artificial tears and lubricating drops treat primarily the aqueous deficiency, while IPL and thermal pulsation therapy treat meibomian gland dysfunction. Multiple treatments are then needed to adequately manage most patients suffering from DES.

Neurostimulation is a novel therapy that takes advantage of the trigeminal nerve pathways that stimulate the lacrimal functional unit. The lacrimal functional unit is defined as the lacrimal glands, ocular surface (cornea, conjunctiva, and meibomian glands), and eyelids as well as the sensory and motor nerves that connect them. It is responsible for regulation, production, and health of the precorneal tear film. ${ }^{8}$ Most commonly the afferent signal comes through nociceptors on the cornea and ocular surface passing through the trigeminal nerve. However, through the nasolacrimal reflex, intranasal stimulation of the nasal branch of the trigeminal nerve can also produce the same efferent autonomic signals to the meibomian glands, goblet cell, and lacrimal glands. By effectively stimulating all aspects of the lacrimal functional unit, neurostimulation has uniquely been shown in recent studies to improve all aspects of the tear film including aqueous, mucin, and lipid. ${ }^{9}$ Utilizing this alternative pathway, neurostimulation has recently been discovered as a novel therapy for the treatment of DES. ${ }^{10}$ In our study, we sought to analyze the optical quality as well as the tear film quality in response to intranasal neurostimulation in patients with DES.

\section{Patients and Methods}

\section{Study Design and Data Collection}

This study was a single-institution cross-sectional review of all patients at the Duke Eye Center with DES who underwent dry eye evaluation and intranasal neurostimulation (TrueTear, Allergan, Irvine, CA, USA) by a single provider (PKG) between January 1, 2018 and January 1, 2019. The study protocol was approved by the Institutional Review Board, Duke University Hospital, Durham, North
Carolina, USA, and was performed in accordance with the tenets of the Declaration of Helsinki and the US Health Insurance Portability and Accountability Act. A waiver of informed consent was granted due to the retrospective nature of this study.

Patients were identified via a review of electronic medical records. There were 21 patients who met the inclusion criteria; 33 eyes were evaluated with intranasal neurostimulation. All included subjects underwent a complete ophthalmic examination by a cornea specialist (PKG), and all eyes included were analyzed with the tear film analysis software of the double-pass (DP) imaging system (HD Analyzer ${ }^{\mathrm{TM}}$, Visiometrics S.L., Terrassa, Spain) before and after intranasal neurostimulation as a part of routine dry eye evaluation. Patient data were assessed using the institution's electronic medical records. Relevant demographic data, including age, sex, race, and ocular co-morbidities, were collected. The optical quality and tear film quality data before and after intranasal neurostimulation were compared.

Exclusion criteria included age $<22$ years, significantly deviated nasal septum, active sinus infection, inability to tolerate neurostimulation, previous ocular trauma, recent corneal surgery within the last 3 months, active recurrent corneal erosions, active filamentary keratitis, superior limbic keratoconjunctivitis, limbal stem cell deficiency, active herpetic keratitis, or neurotrophic cornea.

After the baseline slit-lamp examination, all patients were brought to a separate patient room for optical and tear film quality assessment before and after neurostimulation. The room was temperature controlled and the lighting was kept uniformly dim throughout testing. After the baseline tear film analysis, a brief intranasal neurostimulation demonstration was given. The patient then applied the intranasal neurostimulation until tear formation occurred or up to 45 seconds. Of note, the device automatically turns off after 60 seconds and the manual recommends no longer than 3 minutes in a single use. The initial neurostimulation level was started at level 2 , and the patient was allowed to adjust the level as needed to produce the desired effect while maintaining an acceptable comfort level. Immediately following neurostimulation, the optical quality and tear film quality were retested using the tear film analysis software of the DP imaging system. The average time to reposition the patient back into in the DP imaging system and begin repeat testing after neurostimulation was roughly 20 seconds. 
To assess the optical quality, we compared both the OSI and MTF before and after neurostimulation. The optical quality as measured by the OSI or MTF is reflective of the entire optical system including the tear film, cornea, aqueous, lens, and vitreous. However, as the intranasal neurostimulation is not changing any of the optical quality components other than tear film it can be used to assess changes in overall optical and tear film quality. To assess the tear film quality, we compared the time to blink and the mean tear film OSI as measured by the tear film analysis software of the DP imaging system before and after neurostimulation. In measuring the time to blink, if the patient blinked within the first 3 seconds then the time of the patient blink was counted as the zero second starting point. In cases where multiple optical quality or tear film assessment was performed either pre or post, the highest quality was used in analysis.

\section{Statistical Analysis}

Statistical analysis was performed with JMP Pro 14 (SAS Institute, Cary, NC) and Stata 16.1 (StataCorp, College Station, TX). Generalized estimating equations were used to compare the pre- and post-treatment values to account for the fact that more than one eye was included in some subjects. Spearman correlations were used to evaluate the strength of association between age and OSI, MTF, tear film mean OSI, and time to blink, as well as the association of OSI with the other optical tear analysis parameters. Wilcoxon rank-sum was used to evaluate the association of gender with each of these factors. A p-value $<0.05$ was considered statistically significant.

\section{Results}

Of the 21 patients evaluated, $81 \%$ were female $(n=17)$ with a mean age of $64.8 \pm 14.9$ years. The majority of patients were Caucasian $(\mathrm{n}=19)$. Of the 19 patients with documented ocular surface index score, the mean was 22.37 (range 6-37). The mean eyelid meibomian gland disease was 2.43 (range 0-4). Eight patients were on cyclosporine and 8 patients were on lifitegrast, while 6 and 5 had failed cyclosporine and lifitegrast, respectively.

\section{Optical Tear Analysis Before Intranasal Neurostimulation}

The pre- and post-intranasal neurostimulation optical tear analysis is demonstrated in Table 1. Age had a statistically
Table I Optical Analyses Pre- and Post-Intranasal Neurostimulation

\begin{tabular}{|l|l|l|l|}
\hline & Pre- & Post- & p-value \\
\hline MTF & $27.67 \pm 11.54$ & $29.79 \pm 10.81$ & 0.15 \\
OSI & $2.08 \pm 1.70$ & $1.78 \pm 1.39$ & 0.015 \\
Tear film OSI & $2.78 \pm 2.01$ & $2.70 \pm 1.99$ & 0.55 \\
Time to blink (s) & $13.94 \pm 6.64$ & $14.52 \pm 6.73$ & 0.44 \\
\hline
\end{tabular}

Note: P-value from generalized estimating equations.

Abbreviations: MTF, modulation transfer function; OSI, objective scatter index.

significant and moderate positive correlation with prestimulation OSI (Spearman's rho 0.60, p <0.001) as demonstrated in Figure 1A, but a smaller negative correlation with pre-stimulation MTF (Spearman's rho -0.38 , $p=0.03$ ) as demonstrated in Figure 1B. Age and prestimulation tear film mean OSI had a moderate positive correlation (Spearman's rho 0.45, $\mathrm{p}=0.009$ ), but it was not correlated with time to blink (Spearman's rho 0.06, $\mathrm{p}=0.72$ ). OSI had a strong negative correlation with MTF prior to neurostimulation (Spearman rho $-0.85, \mathrm{p}<0.001$, Figure 1C), a strong positive correlation with tear film mean OSI (Spearman rho 0.85, p <0.001, Figure 1D), and no correlation with time to blink (Spearman's rho $0.008, \mathrm{p}=0.97$ ). There was no association between gender and pre-stimulation tear film mean OSI or time to blink (both $\mathrm{p}>0.20$ ). However, women had a significantly lower pre-stimulation OSI (1.801.60 vs $2.95 \pm 1.83$, Wilcoxon rank-sum $\mathrm{p}=0.0224$ ) and a significantly higher prestimulation MTF $(30.0 \pm 11.5$ vs 20.48 .7 , Wilcoxon ranksum $\mathrm{p}=0.04$ ) compared to men.

\section{Optical Tear Analysis After Intranasal Neurostimulation}

The average OSI and MTF after intranasal neurostimulation were $1.78 \pm 1.39$ and $29.79 \pm 10.81$, respectively. Thus, the optical quality, as measured by OSI, improved significantly after intranasal neurostimulation with an average decrease (improvement) in OSI of $0.30 \pm 0.68$ $(\mathrm{p}=0.015$; Figure 2). Optical quality as measured by MTF improved after intranasal neurostimulation use, with an average increase (improvement) in MTF of $2.12 \pm 9.2(p=0.15)$; however, this change did not reach statistical significance. Neither tear film OSI nor time to blink changed significantly after neurostimulation (all $\mathrm{p}>0.20$ ).

No adverse effects were observed after intranasal neurostimulation other than some subjective mild discomfort during device use reported by several patients. 

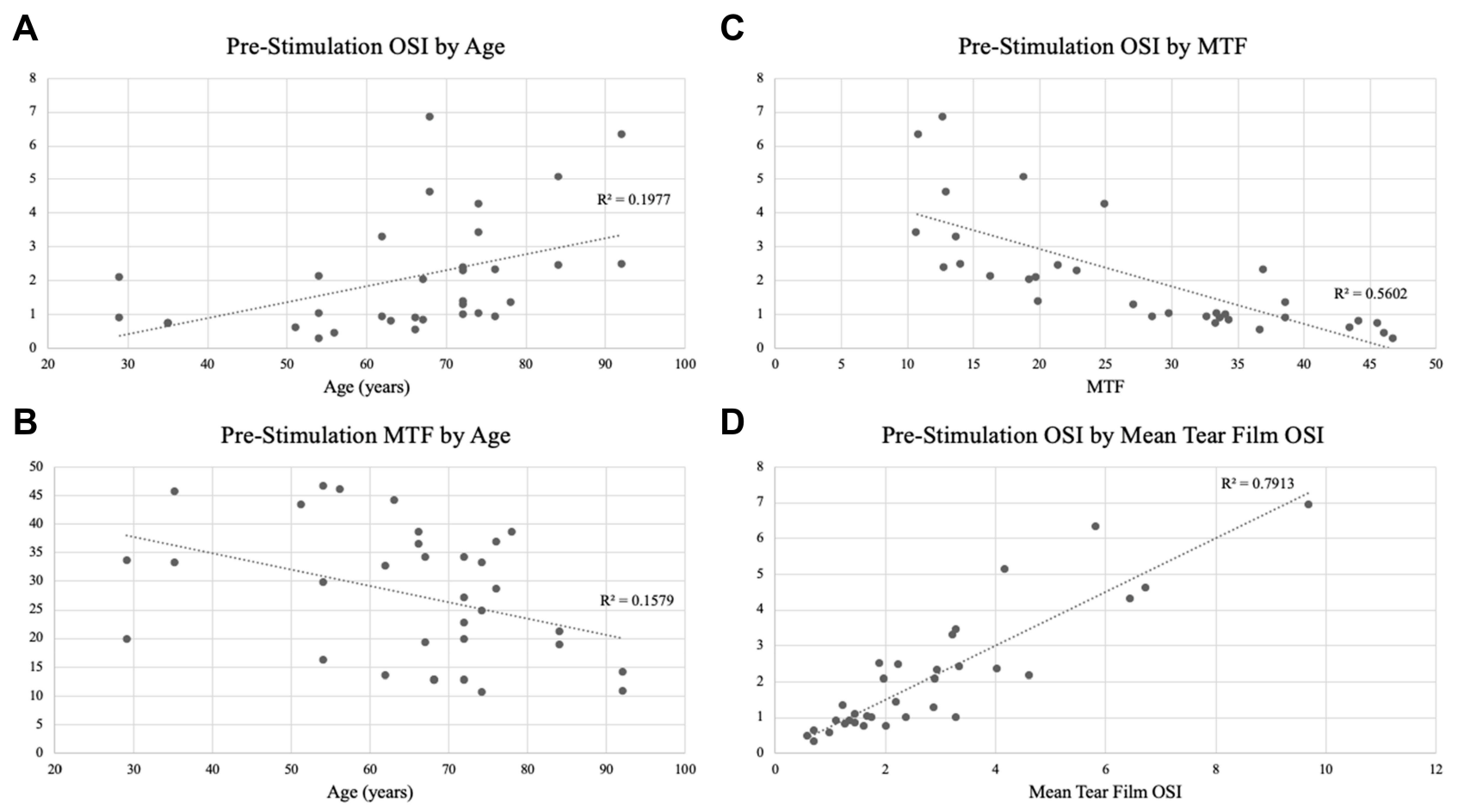

Figure I Graph showing pre-intranasal neurostimulation optical quality correlations with a $(\mathbf{A})$ small positive correlation of OSI with age (Spearman rho 0.60 , $p<0.00 \mathrm{I})$, (B) small negative correlation of MTF with age (Spearman rho $-0.38, p=0.03$ ), (C) strong negative correlation of OSI with MTF (Spearman rho -0.85 , $p<0.00 \mathrm{I}$ ), and (D) strong positive correlation of OSI with tear film mean OSI (Spearman rho 0.85, $\mathrm{p}<0.00 \mathrm{I}$ ).

Abbreviations: OSI, objective scatter index; MTF, modulation transfer function.

\section{Objective Scatter Index (OSI)}

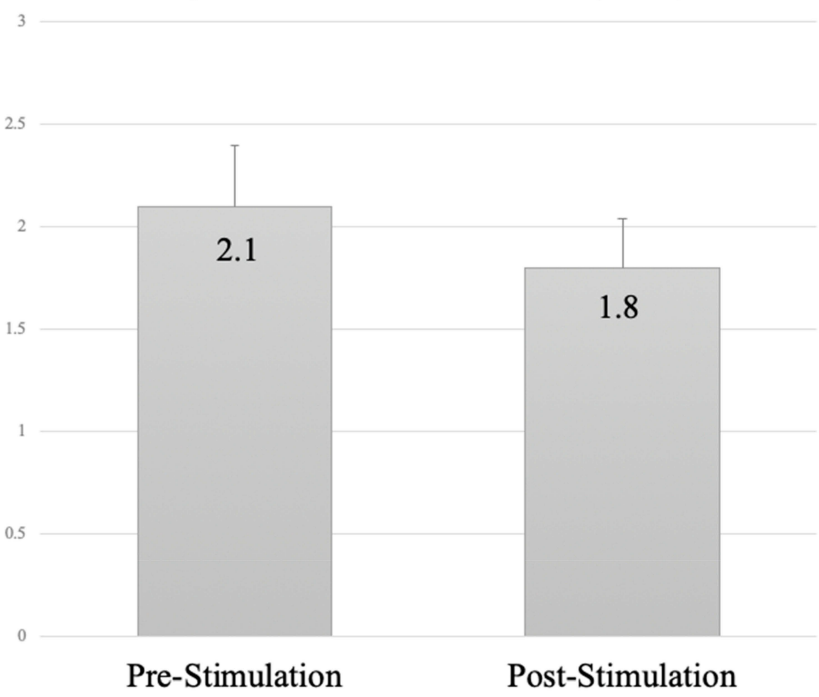

Figure 2 Optical quality analysis as measured by the ocular scatter index (OSI) before and after intranasal neurostimulation $(p=0.015)$.

\section{Discussion}

Overall ocular optical quality depends on the summation of each isolated refractive component of the eye. The variable components of this system largely involve changes in the lens over time and dynamic moment to moment tear film changes. Numerous reports have demonstrated a decrease in optical quality with reduced tear break-up due to conditions such as dry eye. ${ }^{4-6}$ To our knowledge, this is the first study to assess optical and tear film quality with the tear film analysis software of a DP imaging system before and after intranasal neurostimulation.

Major optical quality variables analyzed with tear analysis software include OSI, which measures the amount of light scattered as it passes through the eye, and MTF, which is the relationship between the contrast of an object and is associated image at every spatial frequency. Higher OSI scores are associated with greater scatter, higher ocular surface disease index scores, reduced tear break-up time, lower Schirmer scores, and overall poorer optical quality. ${ }^{11}$ Conversely, a lower MTF, which has also been demonstrated in DES, is associated with decreased contrast sensitivity and increased higher order aberrations. ${ }^{12}$

In our preliminary study we found neurostimulation generated a statistically significant positive impact in patients' OSI, and an overall trend towards improvement in their MTF. This is similar to a prior study by Diaz-Valle et al who analyzed the change in OSI in response to 
lubricant eyedrops in dry-eye patients and found that patients with mild to moderate dry eye had an improved OSI change rate for at least 60 minutes after instillation of lubricating eye drops. ${ }^{13}$ Despite neurostimulation resulting in an improvement in optical quality OSI and MTF, the impact on mean tear film OSI was less impressive. One possible explanation for this finding is that the aqueous, mucin, and lipid components of the natural tear are not proportionally increased in patients with DES concomitant with meibomian gland disease. While the aqueous and mucin components of the tear may be immediately increased, in patients with meibomian gland disease, the improvement in the lipid component of the tear film may be delayed due to blocked and inspissated glands. Following this logic, the improved aqueous/mucin components would improve the optical quality OSI (measured in a few seconds); however, the tears would evaporate quickly with a significant drop off in tear film OSI after several seconds (tear film OSI measured without blink over 20 seconds). While our study only analyzed the optical and tear film quality immediately after initial neurostimulation, it is yet to be determined whether repeated neurostimulation alone could improve meibomian gland function or if complementary therapy such as thermal pulsation or manual expression would be needed as a supplemental treatment.

Our study also demonstrated a positive correlation between pre-intranasal neurostimulation OSI and age and a negative correlation between pre-intranasal neurostimulation MTF and age, which is consistent with existing literature. ${ }^{14,15}$ This is likely multifactorial and related to both an increased prevalence of dry eye and decrease in crystalline lens transparency with increased age. We did not investigate the impact of phakic vs pseudo-phakic status in order to delineate the contribution of the lens on OSI, but this would represent a potential area of future investigation.

Our study was limited by a modest sample size with primarily Caucasian females, a short interval between a single intranasal neurostimulation treatment and repeat optical analysis, and a lack of longitudinal follow up. The degree of dry eye severity amongst our population may have also impacted the statistical significance of pre- and post-intranasal stimulation variables and should be taken into consideration. Furthermore, given the lack of recommendations regarding a specific duration of stimulation, one area of potential future research could involve varying the duration of intra-nasal stimulation. However, we feel this research provides a foundation for future investigation of the effect of neurostimulation on optical and tear film quality in patients with DES.

In conclusion, we found a significant improvement in the OSI of patients with DES immediately following neurostimulation, with a trend toward improvement in MTF. Future investigation is needed to determine the long-term impact of neurostimulation in patients with DES.

\section{Disclosure}

PKG is a consultant to and reports personal fees from Allergan, outside the submitted work. None of the remaining authors have any financial interest in the research product being studied and the authors report no other possible conflicts of interest in this work. This study received no funding.

\section{References}

1. O'Brien PD, Collum LM. Dry eye: diagnosis and current treatment strategies. Curr Allergy Asthma Rep. 2004;4(4):314-319. doi:10.1007/s11882-004-0077-2

2. Schein OD, Munoz B, Tielsch JM, Bandeen-Roche K, West S. Prevalence of dry eye among the elderly. Am J Ophthalmol. 1997;124(6):723-728. doi:10.1016/S0002-9394(14)71688-5

3. Moss SE, Klein R, Klein BE. Long-term incidence of dry eye in an older population. Optom Vis Sci. 2008;85(8):668-674. doi:10.1097/ OPX.0b013e318181a947

4. Montes-Mico R, Alio JL, Charman WN. Dynamic changes in the tear film in dry eyes. Invest Ophthalmol Vis Sci. 2005;46(5):1615-1619. doi:10.1167/iovs.05-0017

5. Montes-Mico R. Role of the tear film in the optical quality of the human eye. J Cataract Refract Surg. 2007;33(9):1631-1635. doi:10.1016/j.jcrs.2007.06.019

6. Xi L, Qin J, Bao Y. Assessment of tear film optical quality in a young short tear break-up time dry eye: case-control study. Medicine (Baltimore). 2019;98(40):e17255. doi:10.1097/MD.0000000000017255

7. Moshirfar M, Pierson K, Hanamaikai K, Santiago-Caban L, Muthappan V, Passi SF. Artificial tears potpourri: a literature review. Clin Ophthalmol. 2014;8:1419-1433. doi:10.2147/OPTH.S65263

8. Weisenthal RW, Afshari NA, Bouchard CS. Structure and Function of the External Eye and Cornea. In: Weisenthal RW. Basic and Clinical Science Course (BCSC): External Disease and Cornea. San Fransisco (CA): American Academy of Ophthalmology; 2015:3-2348.

9. Brinton M, Kossler AL, Patel ZM, et al. Enhanced tearing by electrical stimulation of the anterior ethmoid nerve. Invest Ophthalmol Vis Sci. 2017;58(4):2341-2348. doi:10.1167/iovs.16-21362

10. Dieckmann G, Fregni F, Hamrah P. Neurostimulation in dry eye disease-past, present, and future. Ocul Surf. 2019;17(1):20-27. doi:10.1016/j.jtos.2018.11.002

11. Herbaut A, Liang H, Rabut G, et al. Impact of dry eye disease on vision quality: an optical quality analysis system study. Transl Vis Sci Technol. 2018;7(4):5. doi:10.1167/tvst.7.4.5

12. Denoyer A, Rabut G, Baudouin C. Tear film aberration dynamics and vision-related quality of life in patients with dry eye disease. Ophthalmology. 2012;119(9):1811-1818. doi:10.1016/j.ophtha.2012. 03.004

13. Diaz-Valle D, Arriola-Villalobos P, Garcia-Vidal SE, et al. Effect of lubricating eyedrops on ocular light scattering as a measure of vision quality in patients with dry eye. J Cataract Refract Surg. 2012;38 (7):1192-1197. doi:10.1016/j.jcrs.2012.02.040 
14. Kamiya K, Umeda K, Kobashi H, Shimizu K, Kawamorita T, Uozato $\mathrm{H}$. Effect of aging on optical quality and intraocular scattering using the double-pass instrument. Curr Eye Res. 2012;37 (10):884-888. doi:10.3109/02713683.2012.688164
15. Guirao A, Gonzalez C, Redondo M, Geraghty E, Norrby S, Artal P. Average optical performance of the human eye as a function of age in a normal population. Invest Ophthalmol Vis Sci. 1999;40(1):203-213.
Clinical Ophthalmology

\section{Publish your work in this journal}

Clinical Ophthalmology is an international, peer-reviewed journal covering all subspecialties within ophthalmology. Key topics include: Optometry; Visual science; Pharmacology and drug therapy in eye diseases; Basic Sciences; Primary and Secondary eye care; Patient Safety and Quality of Care Improvements. This journal is indexed on PubMed

Submit your manuscript here: https://www.dovepress.com/clinical-ophthalmology-journal

\section{Dovepress}

Central and CAS, and is the official journal of The Society of Clinical Ophthalmology (SCO). The manuscript management system is completely online and includes a very quick and fair peer-review system, which is all easy to use. Visit http://www.dovepress.com/ testimonials.php to read real quotes from published authors. 